\title{
PRODUCING POTATO VIA NATURAL, ORGANIC AND BIO AMENDMENTS
}

\author{
ABDEL-ATI, Y.Y. ${ }^{1}$, Y.T. ABD EL-MAGEED ${ }^{1}$, M.M. FARRAG ${ }^{1}$, \\ S. ABOU-EL-HASSAN ${ }^{2}$ and B.A.A. SALEM ${ }^{1}$
}

1. Hort. Dept. Fac. of Agric. Minia Univ., Egypt.

2. Central Lab of Organic Agriculture, Agricultural Research Center, Egypt.

(Manuscript received 22 February 2019)

\begin{abstract}
$\mathrm{F}$ ield experiment was performed to evaluate whether using compost and natural rocks of phosphate and potassium with bio fertilizers could replace mineral fertilizers in potato production. The experiment was conducted at Giza Agriculture Research Station, Egypt. The potato tubers (Lady Rosetta and Lady Balfour cultivars) were planted during the third week of January 2016 and 2017 seasons. Individual or combined treatments of rock phosphate with phosphate dissolving bacteria (Bacillus megaterium) and feldspar with $\mathrm{K}$ releasing bacteria (Bacillus circulans) were applied in presence either mineral $\mathrm{N}$ or compost with $\mathrm{N}$ fixing bacteria (Azotobacter chroococcum + Azospirillium brasilense). The effects of these treatments on growth characteristics and yield component of potato were compared with recommended dose of NPK as mineral fertilizers. The results mentioned that Lady Balfour cultivar was significantly better in most vegetative traits and yield component than Lady Rosetta. All individual or combined treatments of rock phosphate and feldspar with mineral $\mathrm{N}$ did not show any significant differences in growth and yield characteristics compared to mineral NPK. All treatments of rock phosphate and feldspar with compost reduced the growth and yield characteristics compared to mineral NPK. The lowest reductions in yield were obtained by compost + rock phosphate and feldspar treatment. In general, rock phosphate and feldspar with phosphate dissolving bacteria and $\mathrm{K}$ releasing bacteria could be an alternative to mineral PK for similar yield and quality of potatoes as well as obtain the highest net return. Compost with $\mathrm{N}$ fixing bacteria could be a substitute for mineral $\mathrm{N}$ to produce a slightly lower yield of potatoes but it's distinguished by high quality and healthy.
\end{abstract}

Keywords: Potato, compost, rock phosphate, feldspar, bio fertilizer, mineral fertilizer alternatives.

\section{INTRODUCTION}

Potato (Solanum tuberosum L.) is one of the most important agricultural crops for world food production after cereal crops, planted with 19,098,328 hectares and produced 381,682,144 tons (FAO 2014). Potato is one of the most important members of solanaceous vegetables in Egypt grown for either local consumption or exportation, it's total cultivated area is about 437386 feddans (feddan $=0.42$ hectare), produced about 4955445 tons (Ministry of Agriculture and Land Reclamation, 2015). The export of potatoes was 650 thousand tons at a value of 241 million US\$ (General Authority for the Control of Exports and Imports, 2015). 
Generally, potato crop requires a huge amount of nitrogen, phosphorus and potassium fertilizer for high productivity and quality. The high prices of chemical fertilizers are currently a major burden for potato farmers and countries that subsidize these fertilizers (Labib et al., 2012). The mineral fertilizers of N, P and K are rapidly lost by either evaporation or leaching in drainage water. The problem does not only stop at losing huge amounts of fertilizers, but it extends to other dangerous environmental pollution (Lee and Song, 2007; Shehata et al., 2014). Slow release forms of $\mathrm{N}, \mathrm{P}$ and $\mathrm{K}$ nutrients include organic nitrogen such as compost, natural minerals of phosphorus and potassium such as rock phosphate and feldspar respectively, these materials are released at a slower rate throughout the season, and thus the plants become able to uptake its most requirements of nutrients throughout the season without lose by leaching. (Abdel-Mouty and El-Greadly, 2008). Organic production of potato using suitable amount of compost could be a substitute to traditional production without decreasing productivity and quality (El-Sayed et al., 2014), with low nitrate content and better storage ability (El-Sayed et al., 2015). Compost, rock phosphate, feldspar and biofertilizers could be an alternative to mineral fertilizer for potato production (Shehata et al., 2014). Other studies reported decreases in plant productivity owing to use organic fertilizer compared to mineral fertilizer (Abou-zeid et al., 2011). Using organic fertilizers for potato production led to produce potatoes with higher content of dry matter and starch than mineral fertilizers. (Abdel-Salam and Shams, 2012). Likewise, decreases in nitrate (Mohammadi et al., 2013; El-Sayed et al., 2015). Utilization of biofertilizers included $\mathrm{N}$ fixing bacteria, phosphate dissolving bacteria and $\mathrm{K}$ releasing bacteria with application of rocks (rock phosphate and feldspar) in soil improve NPK uptake and the yield parameters of plants (Shaaban et al., 2015). Biofertilizer proved to be very effective in increasing potato quality by reducing nitrate content (Shehata et al., 2014). This study aims to investigate the effect of non-chemical fertilizers using compost and natural rocks of $P$ and $\mathrm{K}$ with adding bio fertilizers on the productivity and quality of potato crop.

\section{MATERIALS AND METHODS}

The field experiment was carried out on potato at Giza Agriculture Research Station, Giza Governorate, Egypt, during the summer seasons of 2016 and 2017.

\section{Plant Material}

Potato tubers (Lady Rosetta and Lady Balfour cultivars) were sown on 20 and 18 of January in the first and second seasons, respectively. 


\section{Soil Properties}

The tubers were planted in clay soil and were irrigated using surface irrigation system. The soil was analyzed according to FAO (1980) and its physical and chemical properties were presented in Table 1.

Table 1. Physical and chemical properties of the experimental soil

\begin{tabular}{|c|c|c|c|c|c|c|c|c|c|c|c|c|c|}
\hline \multirow{2}{*}{$\begin{array}{c}\text { Sand } \\
\%\end{array}$} & $\begin{array}{c}\text { Silt } \\
\%\end{array}$ & $\begin{array}{c}\text { Clay } \\
\%\end{array}$ & Texture & $\mathrm{pH}$ & $\begin{array}{c}\mathrm{EC} \\
\mathrm{dS} / \mathrm{m}\end{array}$ & \multicolumn{4}{|c|}{ Cations meq/l } & \multicolumn{4}{|c|}{ Anions meq/l } \\
\cline { 6 - 12 } & & & $\mathrm{Ca}^{++}$ & $\mathrm{Mg}^{++}$ & $\mathrm{K}^{+}$ & $\mathrm{Na}^{+}$ & $\mathrm{CO}_{3}=$ & $\mathrm{HCO}_{3}^{-}$ & $\mathrm{Cl}^{-}$ & $\mathrm{SO}_{4}=$ \\
\hline 23.9 & 36.6 & 39.5 & Clay loam & 7.59 & 2.45 & 5.20 & 2.80 & 0.80 & 10.35 & - & 0.76 & 16.23 & 1.80 \\
\hline
\end{tabular}

\section{The Experimental Layout}

The experiment soil was ploughed and divided into ridges $(70 \mathrm{~cm}$ width). The tubers were sown at a distance of $20 \mathrm{~cm}$ on one side of ridge.

\section{The Experimental Treatments}

1- Mineral fertilizers of NPK (MNPK) as a control

2- Mineral fertilizers of NP + feldspar with $K$ releasing bacteria (MNP + F \& KRB)

3- Mineral fertilizers of NK + rock phosphate with $\mathrm{P}$ dissolving bacteria (MNP + RP \& PDB)

4- Mineral fertilizers of $\mathrm{N}+$ rock phosphate with $\mathrm{P}$ dissolving bacteria + feldspar with $K$ releasing bacteria $(M N+R P \& P D B+F \& K R B)$

5- Compost (C) with $\mathrm{N}$ fixing bacteria + MPK (C \& NFB + MPK)

6- $C \& N F B+M P+F \& K R B$

7- $C \& N F B+M K+R P \& P D B$

8- $C \& N F B+R P \& P D B+F \& K R B$

\section{Experimental Design}

The experiments were arranged in split plot design with using three replicates. The potato cultivars were adapted in the main plots and the fertilizer treatments were randomized in the sub plots. The plot area was $10.5 \mathrm{~m}^{2}$ ( $3 \mathrm{~m}$ length and $3.5 \mathrm{~m}$ width). Each plot included 5 ridges.

\section{Quantities of application}

The mineral fertilizers of NPK were applied as follow $150 \mathrm{~kg} \mathrm{~N} / \mathrm{fed}$ as $448 \mathrm{~kg}$ ammonium nitrate $(33.5 \% \mathrm{~N}), 60 \mathrm{~kg} \mathrm{P}_{2} \mathrm{O}_{5} / \mathrm{fed}$ as $387 \mathrm{~kg}$ calcium super phosphate $\left(15.5 \% \mathrm{P}_{2} \mathrm{O}_{5}\right)$ and $96 \mathrm{~kg} \mathrm{~K} \mathrm{~K}_{2} \mathrm{O} /$ fed as $200 \mathrm{~kg}$ potassium sulphate $\left(48 \% \mathrm{~K}_{2} \mathrm{O}\right)$. The quantity of compost was calculated based on nitrogen recommended dose in clay soil (150 kg/feddan); that was 16.5 tons/feddan as dry weight. Analyses of the used compost were analyzed according to FAO (1980) and showed in Table 2. Rock phosphate $\left(20 \% \mathrm{P}_{2} \mathrm{O}_{5}\right)$ and feldspar $\left(10 \% \mathrm{~K}_{2} \mathrm{O}\right)$ were applied at rate 300 and 960 $\mathrm{kg} / \mathrm{fed}$ on respectively. Nitrogen fixing bacteria was used as mixture of Azotobacter chroococcum and Azospirillium brasilense. Phosphate dissolving bacteria and potassium releasing bacteria were used as single strains of Bacillus megaterium and Bacillus circulans on respectively. 
Table 2. Analyses of the used compost

\begin{tabular}{|c|c|c|c|c|c|c|c|c|c|}
\hline \multirow{2}{*}{ pH 1:10 } & \multirow{2}{*}{$\begin{array}{c}\text { EC } 1: 10 \\
\mathrm{dS} / \mathrm{m}\end{array}$} & \multirow{2}{*}{$\begin{array}{l}\text { O.C } \\
(\%)\end{array}$} & \multirow{2}{*}{$\begin{array}{l}\text { O.M } \\
(\%)\end{array}$} & \multirow{2}{*}{$\begin{array}{l}\mathrm{C} / \mathrm{N} \\
\text { Ratio }\end{array}$} & $\mathrm{N}$ & $\mathrm{P}_{2} \mathrm{O}_{5}$ & $\mathrm{~K}_{2} \mathrm{O}$ & $\mathrm{N}-\mathrm{NH}_{4}$ & $\mathrm{~N}-\mathrm{NO}_{3}$ \\
\hline & & & & & \multicolumn{3}{|c|}{ (\%) } & \multicolumn{2}{|c|}{ ppm } \\
\hline 7.12 & 3.69 & 17.15 & 28.13 & 18.84 & 0.91 & 0.71 & 1.20 & 302.00 & 76.00 \\
\hline
\end{tabular}

\section{Time and Method Application}

All quantities of calcium super phosphate, compost, rock phosphate and feldspar were added as one dose during soil preparation, whereas ammonium nitrate and potassium sulphate were added at two equal portions after 30 and 50 days from sowing. All bio fertilizers were supplemented to the soil surface beside plants at rate 5 L/fed after 20 and 40 days from sowing by using liquid cultures ( $1 \mathrm{ml}$ contains $10^{8} \mathrm{cell}$ ) according to Mashhoor et al. (2002).

\section{Data Recorded}

\section{Growth characteristics and nutritional status}

After 75 days from sowing, six plants were randomly sampled from the inner 3 rows of each plot to determine parameters of vegetative growth and nutritional status. Fresh weight of shoot, plant height, numbers of stems and leaves per plant were measured. Also, chlorophyll reading was recorded in the third upper leaf by using Minolta Chlorophyll Meter Spad 501. Nutrient content (NPK) in potato plants were determined in dry matter of the third upper leaf according to Cottenie et al. (1982). Total nitrogen, phosphorus and potassium were determined by Micro Kjeldahl, Spectrophotometer and Flame photometer on respectively according to FAO (1980).

\section{Yield component and quality properties}

Potato tubers were harvested at maturity stage after 110 and 120 days from sowing for Lady Rosetta and Lady Balfour cultivars respectively. Five plants from each experimental unit were randomly chosen to measure weight and number of tubers per plant. Total yield/fed. was calculated from plot yield. Potato tubers were graded to three sizes small $(2.5-5 \mathrm{~cm})$, medium $(5.0-6.5 \mathrm{~cm})$ and large $(>6.5 \mathrm{~cm})$ according to Adams and Hide (1981) and the percentage of each size per meter square were calculated. Ten tubers from each replicate were randomly sampled to determine specific gravity and dry matter in tubers. Total soluble solids (TSS) in tubers were measured by using Digital Refractometer. Percentage of starch and carbohydrate were determined according to AOAC (2005). As well as nitrate content of tubers was determined using Cardy Nitrate Meter Model HORIBA, Spectrum Technologies, Inc., as described by Al-Moshileh et al. (2004).

\section{Economic evaluate}

Economic evaluate was performed by estimate the net return of studied treatments. Cost of cultivation was calculated as sum costs of land preparation, irrigation, fertilization, pest management, transportation, land rent, seeding price and 
other expenses based on local charges for all cultivars and treatments (Table 3). Gross return was obtained as the sum price of the total yield at harvesting time on the basis of local field price. Net return was calculated by subtracting total cost of cultivation from gross return.

Table 3. Total cost cultivation of two potato cultivars as mean for both seasons (2016 and 2017)

\begin{tabular}{|c|c|c|c|c|c|c|}
\hline \multirow{2}{*}{\begin{tabular}{l}
\multicolumn{1}{c}{ Items } \\
Seeding \\
Soil \\
preparation
\end{tabular}} & \multirow{2}{*}{$\begin{array}{c}\text { Cost } \\
\text { (L.E./fed.) } \\
7500 \\
600\end{array}$} & \multirow{2}{*}{$\begin{array}{l}\text { Fertilizer } \\
\text { treatments }\end{array}$} & \multirow{2}{*}{$\begin{array}{l}\text { Fertilizer } \\
\text { cost } \\
\text { (L.E./fed.) }\end{array}$} & \multirow{2}{*}{$\begin{array}{l}\text { Production } \\
\text { cost } \\
\text { (L.E./fed.) }\end{array}$} & \multicolumn{2}{|c|}{$\begin{array}{l}\text { Total cost } \\
\text { (L.E./fed.) }\end{array}$} \\
\hline & & & & & Rosseta & Balfour \\
\hline Irrigation & 400 & MNPK & 3447 & 15100 & 18547 & 18547 \\
\hline Weeding & 600 & $M N P+F$ & 2715 & 15100 & 17815 & 17815 \\
\hline Pest control & 1000 & MNK + RP & 3284 & 15100 & 18384 & 18384 \\
\hline Harvesting & 600 & $M N+R P+F$ & 2552 & 15100 & 17652 & 17652 \\
\hline Transportation & 400 & $C+M P K$ & 5503 & 15100 & 20603 & 20603 \\
\hline $\begin{array}{l}\text { Other } \\
\text { expenses }\end{array}$ & 1000 & $C+M P+F$ & 4771 & 15100 & 19871 & 19871 \\
\hline Rent & 3000 & $C+R P+M K$ & 5340 & 15100 & 20440 & 20440 \\
\hline Production cost & 15100 & $C+R P+F$ & 4608 & 15100 & 19708 & 19708 \\
\hline
\end{tabular}

\section{Statistical analysis}

Data of both seasons were arranged and statistically analyzed according to Snedecor and Cochran (1980) with SAS software, version 2004. Treatment means were compared at significance level 0.05 using Tukey test.

\section{RESULTS AND DISCUSSION}

\section{Vegetative growth}

Vegetative growth parameters, i.e. chlorophyll reading in leaves, shoot fresh weight, stem number, plant height and leaf number/plant for two cultivars of potato under different fertilizers were presented in Tables 4 and 5. Data indicated that cv. Lady Balfour showed a significant superiority in all vegetable growth characteristics except chlorophyll reading of leaves and stem number/plant. No significant differences were observed between the two cultivars in stem number/plant. While, Lady Rosetta excelled in chlorophyll reading of leaves. Differences between cultivars might be related to genetic factors which resulted from genetic makeup relations for the cultivars as reported by Zelelew et al. (2016).

Effect of different fertilizers on vegetative growth of potato plants, the results revealed that all mineral nitrogen treatments with the addition of PK in the form of minerals or rocks increased the vegetative growth characteristics of plants compared with compost treatments except for the number of stems, which showed no significant differences among all fertilizer treatments. No significant differences were found among all mineral nitrogen treatments, with addition of PK in the form of minerals or 
rocks. All compost treatments, with adding PK in the form of minerals or rocks, showed no significant differences among them in all vegetable growth characteristics. These results were similar in both seasons. This superiority in mineral nitrogen treatments in vegetative growth properties over compost treatments, might be attributed to the plants obtain nitrogen from mineral fertilizer more easily than organic fertilizer. Nitrogen has an important role in the formation of chlorophyll and growth hormones into plant, which reflect positive effect on vegetative growth These results are compatible with that obtained by Abou-Hussein 2005 and El-Sayed et al. (2014).

Table 4. Effect of treatments on vegetative growth characteristics of two potato cultivars during 2016 and 2017 seasons

\begin{tabular}{|c|c|c|c|c|c|c|c|c|c|c|c|c|}
\hline \multirow{4}{*}{$\begin{array}{l}\text { Fertilizer } \\
\text { treatments }\end{array}$} & \multicolumn{6}{|c|}{ First season } & \multicolumn{6}{|c|}{ Second season } \\
\hline & \multicolumn{12}{|c|}{ Cultivars } \\
\hline & \multicolumn{2}{|c|}{ Rosetta } & \multicolumn{2}{|c|}{ Balfour } & \multicolumn{2}{|c|}{ Mean } & \multicolumn{2}{|c|}{ Rosetta } & \multicolumn{2}{|c|}{ Balfour } & \multicolumn{2}{|c|}{ Mean } \\
\hline & \multicolumn{12}{|c|}{ Chlorophyll reading (SPAD) } \\
\hline MNPK & 43.89 & $a b$ & 40.78 & $\mathrm{bc}$ & 42.33 & A & 51.34 & $a$ & 46.62 & bcd & 48.98 & A \\
\hline$M N P+F$ & 44.89 & $a$ & 40.78 & $b c$ & 42.83 & A & 52.34 & $a$ & 46.62 & bcd & 49.48 & A \\
\hline $\mathrm{MNK}+\mathrm{RP}$ & 44.56 & a & 41.00 & $\mathrm{bc}$ & 42.78 & A & 52.01 & $a$ & 46.84 & b & 49.42 & A \\
\hline$M N+R P+F$ & 45.22 & $a$ & 41.89 & $a b c$ & 43.56 & A & 52.67 & $a$ & 47.73 & $b$ & 50.20 & A \\
\hline $\mathrm{C}+\mathrm{MPK}$ & 40.11 & $\mathrm{~cd}$ & 38.67 & $\mathrm{~cd}$ & 39.39 & B & 45.76 & bcd & 43.14 & def & 44.45 & B \\
\hline$C+M P+F$ & 40.67 & $c d$ & 37.11 & $d$ & 38.89 & B & 44.54 & $b-f$ & 41.58 & $f$ & 43.06 & B \\
\hline$C+R P+M K$ & 39.33 & $\mathrm{~cd}$ & 37.22 & $d$ & 38.28 & B & 44.98 & $b-e$ & 41.69 & ef & 43.34 & B \\
\hline$C+R P+F$ & 40.67 & $b c$ & 39.00 & $\mathrm{~cd}$ & 39.84 & B & 46.32 & $\mathrm{bcd}$ & 43.47 & $c-f$ & 44.90 & $\mathrm{~B}$ \\
\hline Mean & 42.42 & $\mathrm{~A}$ & 39.56 & $\mathrm{~B}$ & & & 48.74 & $\mathrm{~A}$ & 44.71 & $\mathrm{~B}$ & & \\
\hline \multicolumn{13}{|c|}{ Shoot fresh weight ( $\mathrm{g}$ ) } \\
\hline MNPK & 183.67 & cde & 287.67 & $a$ & 235.67 & A & 219.67 & $d$ & 304.67 & $a b$ & 262.17 & A \\
\hline$M N P+F$ & 183.00 & cde & 284.67 & a & 233.84 & A & 214.67 & $d$ & 304.33 & $a b$ & 259.50 & A \\
\hline $\mathrm{MNK}+\mathrm{RP}$ & 184.33 & $\mathrm{~cd}$ & 285.67 & $a$ & 235.00 & A & 220.33 & $d$ & 306.00 & $a$ & 263.17 & A \\
\hline$M N+R P+F$ & 186.67 & c & 291.67 & a & 239.17 & A & 222.67 & $d$ & 308.00 & $a$ & 265.34 & A \\
\hline$C+M P K$ & 148.00 & $f$ & 250.00 & b & 199.00 & B & 177.00 & e & 277.67 & $b c$ & 227.33 & B \\
\hline$C+M P+F$ & 151.67 & def & 247.33 & $b$ & 199.50 & B & 180.67 & e & 273.00 & c & 226.83 & B \\
\hline$C+R P+M K$ & 149.67 & ef & 243.33 & $b$ & 196.50 & B & 178.67 & e & 275.67 & c & 227.17 & B \\
\hline$C+R P+F$ & 148.00 & $f$ & 250.00 & $\mathrm{~b}$ & 199.00 & B & 177.00 & $\mathrm{e}$ & 276.67 & $\mathrm{c}$ & 226.83 & $B$ \\
\hline Mean & 166.88 & B & 267.54 & $\mathrm{~A}$ & & & 198.83 & $B$ & 290.75 & A & & \\
\hline \multicolumn{13}{|c|}{ Stem Number } \\
\hline MNPK & 2.11 & $a$ & 2.33 & $a$ & 2.33 & A & 2.67 & $a$ & 2.33 & $a$ & 2.67 & A \\
\hline$M N P+F$ & 2.11 & $a$ & 2.55 & $a$ & 2.34 & A & 2.67 & a & 3.00 & a & 2.83 & A \\
\hline$M N K+R P$ & 2.22 & $a$ & 2.53 & $a$ & 2.38 & A & 2.67 & a & 2.67 & $a$ & 2.67 & A \\
\hline$M N+R P+F$ & 2.33 & $a$ & 2.44 & a & 2.28 & A & 3.00 & $a$ & 2.67 & a & 2.67 & A \\
\hline$C+M P K$ & 2.11 & $a$ & 2.22 & $a$ & 2.17 & A & 3.00 & $a$ & 2.67 & $a$ & 2.83 & A \\
\hline$C+M P+F$ & 2.33 & $a$ & 2.22 & $a$ & 2.28 & A & 3.33 & $a$ & 2.67 & $a$ & 3.00 & A \\
\hline$C+R P+M K$ & 2.33 & $a$ & 2.22 & $a$ & 2.28 & A & 2.67 & a & 2.33 & $a$ & 2.50 & A \\
\hline$C+R P+F$ & 2.11 & a & 2.22 & a & 2.17 & A & 2.33 & a & 2.67 & $\mathrm{a}$ & 2.50 & $\mathrm{~A}$ \\
\hline Mean & 2.21 & A & 2.34 & $\mathrm{~A}$ & & & 2.79 & A & 2.63 & A & & \\
\hline
\end{tabular}

Means followed in same column by similar letters are not statistically different at 0.05 level according to Tukey test.

MNPK = mineral NPK

$\mathrm{C}=$ compost $+\mathrm{N}$ fixing bacteria

$\mathrm{F}=$ feldspar $+\mathrm{K}$ releasing bacteria

$\mathrm{RP}=$ rock phosphate $+\mathrm{P}$ dissolving bacteria 
The interaction between fertilizer treatments and cultivars had significant effect on vegetative growth characteristics of potato plants in both seasons. However, the highest values of plant height, leaf number and shoot fresh weight/plant were recorded by all mineral $\mathrm{N}$ treatments with $\mathrm{cv}$. Lady Balfour. Meanwhile, the lowest values were obtained by all compost treatments with cv. Lady Rosetta. On contrarily, all compost treatments with Rosetta cultivar gave the highest values of chlorophyll reading of leaves. Whereas, the lowest values were resulted by all mineral $\mathrm{N}$ treatments with cv. Lady Balfour. This might be related to genetic factors resulted from genetic makeup relations for the cultivars.

Table 5. Effect of treatments on vegetative growth characteristics of two potato cultivars during 2016 and 2017 seasons

\begin{tabular}{|c|c|c|c|c|c|c|c|c|c|c|c|c|}
\hline \multirow{3}{*}{$\begin{array}{l}\text { Fertilizer } \\
\text { treatments }\end{array}$} & \multicolumn{6}{|c|}{ First season } & \multicolumn{6}{|c|}{ Second season } \\
\hline & \multicolumn{12}{|c|}{ Cultivars } \\
\hline & \multicolumn{2}{|c|}{ Rosetta } & \multicolumn{2}{|c|}{ Balfour } & \multicolumn{2}{|c|}{ Mean } & \multicolumn{2}{|c|}{ Rosetta } & \multicolumn{2}{|c|}{ Balfour } & \multicolumn{2}{|c|}{ Mean } \\
\hline & \multicolumn{12}{|c|}{ Plant height $(\mathrm{cm})$} \\
\hline MNPK & 49.78 & $b$ & 57.13 & $a$ & 53.46 & $A$ & 51.33 & de & 60.56 & $\mathrm{a}$ & 55.95 & A \\
\hline$M N P+F$ & 50.11 & $b$ & 56.57 & a & 53.34 & A & 51.33 & de & 60.00 & $a$ & 55.67 & A \\
\hline$M N K+R P$ & 49.66 & $b$ & 57.35 & $a$ & 53.51 & A & 50.00 & ef & 60.78 & $a$ & 55.39 & A \\
\hline$M N+R P+F$ & 51.45 & $b$ & 58.35 & $a$ & 54.90 & A & 52.33 & cde & 61.78 & $a$ & 57.06 & A \\
\hline$C+M P K$ & 43.89 & c & 50.11 & $b$ & 47.00 & B & 45.67 & $g$ & 55.11 & bcd & 50.39 & $B C$ \\
\hline$C+M P+F$ & 44.67 & c & 48.89 & $b$ & 46.78 & B & 47.33 & $\mathrm{fg}$ & 53.89 & bcd & 50.61 & C \\
\hline$C+R P+M K$ & 43.00 & c & 50.45 & $b$ & 46.72 & B & 45.33 & $g$ & 55.45 & bcd & 50.39 & $B C$ \\
\hline$C+R P+F$ & 43.89 & $\mathrm{c}$ & 51.11 & $\mathrm{~b}$ & 47.50 & B & 47.67 & $\mathrm{fg}$ & 56.11 & $\mathrm{~b}$ & 51.89 & $\mathrm{~B}$ \\
\hline \multirow[t]{2}{*}{ Mean } & 47.06 & B & 53.74 & A & & & 48.88 & B & 57.96 & A & & \\
\hline & \multicolumn{12}{|c|}{ Leaf number/plant } \\
\hline MNPK & 46.67 & $b c$ & 63.67 & a & 55.17 & $A$ & 52.33 & bcd & 75.33 & $a$ & 63.83 & A \\
\hline$M N P+F$ & 46.00 & $\mathrm{bc}$ & 64.00 & a & 55.00 & A & 51.67 & b-e & 73.67 & a & 62.67 & A \\
\hline MNK + RP & 46.00 & $\mathrm{bc}$ & 66.33 & a & 56.17 & A & 52.33 & bcd & 76.33 & $a$ & 64.33 & A \\
\hline$M N+R P+F$ & 47.33 & $b$ & 64.00 & $a$ & 55.67 & A & 52.67 & $b c$ & 78.33 & a & 65.50 & A \\
\hline $\mathrm{C}+\mathrm{MPK}$ & 39.33 & bc & 44.33 & bc & 41.83 & B & 44.67 & cde & 55.33 & $b$ & 50.00 & B \\
\hline$C+M P+F$ & 39.33 & $b c$ & 43.67 & $b c$ & 41.50 & B & 43.33 & $\mathrm{e}$ & 54.67 & $b$ & 49.00 & B \\
\hline$C+R P+M K$ & 38.00 & c & 43.00 & bc & 40.50 & B & 44.67 & cde & 54.00 & $b$ & 49.33 & B \\
\hline$C+R P+F$ & 39.00 & $\mathrm{bc}$ & 45.00 & $\mathrm{bc}$ & 42.00 & B & 43.67 & de & 56.00 & $b$ & 49.83 & $B$ \\
\hline Mean & 42.71 & B & 54.25 & $\mathrm{~A}$ & & & 47.67 & B & 65.67 & A & & \\
\hline
\end{tabular}

Means followed in same column by similar letters are not statistically different at 0.05 level according to Tukey test.

$\mathrm{MNPK}=$ mineral NPK

$\mathrm{F}=$ feldspar $+\mathrm{K}$ releasing bacteria

$\mathrm{C}=$ compost $+\mathrm{N}$ fixing bacteria

$\mathrm{RP}=$ rock phosphate $+\mathrm{P}$ dissolving bacteria

\section{Nutritional status}

Data in Table 6 indicated that NPK content in leaves of Lady Balfour cultivar was significantly higher than Lady Rosetta in both seasons. These results might be correlated with the gene action of the tested cultivars (Zelelew et al., 2016).

Fertilizer treatments showed significant effect on NK content of potato leaves, whereas there were no effects on $\mathrm{P}$ content in both seasons. The highest $\mathrm{N}$ content was obtained by plants that fertilized by mineral $\mathrm{N}+$ rocks of $\mathrm{PK}+\mathrm{P}$ dissolving and $\mathrm{K}$ releasing bacteria, with no 
significant differences with mineral NPK treatment. Meanwhile, compost with $\mathrm{N}$ fixing bacteria + rocks of $\mathrm{PK}+\mathrm{P}$ dissolving and $\mathrm{K}$ releasing bacteria gave the highest $\mathrm{K}$ content compared to mineral NPK, with no significant differences with other compost treatments. The high content of $\mathrm{N}$ in potato leaves with treatments of mineral $\mathrm{N}$ fertilizer might be due to it is easy decomposition, so the plants absorb large quantities from it. While, superiority of compost + rocks of $\mathrm{P}$ and $\mathrm{K}$ in presence of bio fertilizers treatments in $\mathrm{K}$ content, may be due to the role of bacteria in releasing $\mathrm{K}$ from feldspar (Sheng et al., 2002), as well as the role of compost as organic matter is characterized by a high cation exchange capacity preserves the nutrients without loss by leaching, so plants can uptake them as needed (Abdel-Mouty and El-Greadly, 2008; Fiorentino and Fagnano, 2011).

Table 6. Effect of treatments on nutritional status of two potato cultivars during 2016 and 2017 seasons

\begin{tabular}{|c|c|c|c|c|c|c|}
\hline \multirow{4}{*}{$\begin{array}{l}\text { Fertilizer } \\
\text { treatments }\end{array}$} & \multicolumn{3}{|c|}{ First season } & \multicolumn{3}{|c|}{ Second season } \\
\hline & \multicolumn{6}{|c|}{ Cultivars } \\
\hline & Rosetta & Balfour & Mean & Rosetta & Balfour & Mean \\
\hline & \multicolumn{6}{|c|}{$\% \mathrm{~N}$} \\
\hline MNPK & $4.253 b$ & $4.493 \mathrm{a}$ & $4.373 \mathrm{~A}$ & $4.317 b$ & $4.573 a$ & $4.445 \mathrm{~A}$ \\
\hline$M N P+F$ & $4.143 b$ & $4.270 b$ & $4.207 \mathrm{~B}$ & $4.213 b$ & $4.347 b$ & $4.280 \mathrm{~B}$ \\
\hline MNK + RP & $4.160 \mathrm{~b}$ & $4.280 \mathrm{~b}$ & $4.220 B$ & $4.230 \mathrm{~b}$ & $4.357 b$ & $4.293 B$ \\
\hline$M N+R P+F$ & $4.290 b$ & $4.550 \mathrm{a}$ & $4.420 \mathrm{~A}$ & $4.363 b$ & $4.627 a$ & $4.495 \mathrm{~A}$ \\
\hline $\mathrm{C}+\mathrm{MPK}$ & $3.177 f$ & $3.337 \mathrm{de}$ & $3.257 \mathrm{D}$ & $3.333 \mathrm{e}$ & $3.437 \mathrm{de}$ & $3.385 \mathrm{D}$ \\
\hline$C+M P+F$ & 3.243 def & 3.303 def & $3.273 \mathrm{D}$ & $3.350 \mathrm{e}$ & $3.410 \mathrm{de}$ & $3.380 \mathrm{D}$ \\
\hline$C+R P+M K$ & 3.287 def & 3.437 def & $3.362 \mathrm{D}$ & $3.380 \mathrm{e}$ & $3.543 d$ & $3.462 \mathrm{D}$ \\
\hline$C+R P+F$ & $3.353 \mathrm{de}$ & $3.743 c$ & $3.548 \mathrm{C}$ & $3.483 \mathrm{de}$ & $3.873 \mathrm{~cd}$ & $3.678 \mathrm{C}$ \\
\hline \multirow[t]{2}{*}{ Mean } & $3.738 \mathrm{~B}$ & $3.927 \mathrm{~A}$ & & $3.834 \mathrm{~B}$ & $4.021 \mathrm{~A}$ & \\
\hline & \multicolumn{6}{|c|}{$\% \mathrm{P}$} \\
\hline MNPK & $0.338 b$ & $0.418 a$ & $0.378 \mathrm{~A}$ & $0.408 \mathrm{c}$ & $0.486 a b$ & $0.447 \mathrm{~A}$ \\
\hline$M N P+F$ & $0.335 b$ & $0.419 a$ & $0.377 \mathrm{~A}$ & $0.402 c$ & $0.496 a b$ & $0.445 \mathrm{~A}$ \\
\hline $\mathrm{MNK}+\mathrm{RP}$ & $0.344 b$ & $0.433 a$ & $0.388 \mathrm{~A}$ & $0.408 c$ & $0.499 a$ & $0.454 \mathrm{~A}$ \\
\hline$M N+R P+F$ & $0.351 b$ & $0.433 a$ & $0.392 \mathrm{~A}$ & $0.415 \mathrm{c}$ & $0.490 a b$ & $0.453 \mathrm{~A}$ \\
\hline $\mathrm{C}+\mathrm{MPK}$ & $0.349 b$ & $0.440 a$ & $0.394 \mathrm{~A}$ & $0.427 c$ & $0.494 a$ & $0.460 \mathrm{~A}$ \\
\hline$C+M P+F$ & $0.351 b$ & $0.429 a$ & $0.390 \mathrm{~A}$ & $0.429 c$ & $0.496 a$ & $0.463 \mathrm{~A}$ \\
\hline $\mathrm{C}+\mathrm{RP}+\mathrm{MK}$ & $0.356 b$ & $0.443 a$ & $0.400 \mathrm{~A}$ & 0.434 bc & $0.496 a$ & $0.465 \mathrm{~A}$ \\
\hline$C+R P+F$ & $0.365 b$ & $0.452 a$ & $0.408 \mathrm{~A}$ & $0.435 b c$ & $0.501 \mathrm{a}$ & $0.468 \mathrm{~A}$ \\
\hline \multirow[t]{2}{*}{ Mean } & $0.349 \mathrm{~B}$ & $0.433 \mathrm{~A}$ & & $0.420 \mathrm{~B}$ & $0.495 \mathrm{~A}$ & \\
\hline & \multicolumn{6}{|c|}{$\% \mathrm{~K}$} \\
\hline MNPK & $5.560 \mathrm{~g}$ & $6.152 \mathrm{de}$ & $5.856 \mathrm{C}$ & $5.627 \mathrm{fg}$ & $6.230 \mathrm{~cd}$ & $5.927 \mathrm{C}$ \\
\hline$M N P+F$ & $5.620 \mathrm{fg}$ & $6.171 \mathrm{de}$ & $5.895 \mathrm{C}$ & $5.683 \mathrm{fg}$ & $6.247 \mathrm{~cd}$ & $5.964 \mathrm{C}$ \\
\hline MNK + RP & $5.531 \mathrm{~g}$ & $6.169 \mathrm{de}$ & $5.850 \mathrm{C}$ & $5.573 \mathrm{~g}$ & $6.247 \mathrm{~cd}$ & $5.909 C$ \\
\hline$M N+R P+F$ & $5.625 \mathrm{fg}$ & 6.185 cde & $5.905 \mathrm{BC}$ & $5.697 \mathrm{fg}$ & $6.273 \mathrm{~cd}$ & $5.985 B C$ \\
\hline $\mathrm{C}+\mathrm{MPK}$ & $5.810 \mathrm{fg}$ & $6.288 \mathrm{bcd}$ & $6.049 \mathrm{ABC}$ & $5.910 \mathrm{ef}$ & $6.507 b c$ & $6.208 \mathrm{AB}$ \\
\hline$C+M P+F$ & $5.800 \mathrm{fg}$ & $6.497 a b c$ & $6.148 \mathrm{AB}$ & 5.900 ef & $6.597 a b$ & $6.248 \mathrm{~A}$ \\
\hline$C+R P+M K$ & $5.780 \mathrm{fg}$ & $6.533 a b$ & $6.157 A B$ & $5.893 \mathrm{ef}$ & $6.633 a b$ & $6.263 \mathrm{~A}$ \\
\hline$C+R P+F$ & $5.916 \mathrm{ef}$ & $6.660 \mathrm{a}$ & $6.288 \mathrm{~A}$ & $6.017 \mathrm{de}$ & $6.830 a$ & $6.423 \mathrm{~A}$ \\
\hline Mean & $5.705 \mathrm{~B}$ & $6.332 \mathrm{~A}$ & & $5.788 \mathrm{~B}$ & $6.445 \mathrm{~A}$ & \\
\hline
\end{tabular}

Means followed in same column by similar letters are not statistically different at 0.05 level according to Tukey test.
MNPK $=$ mineral NPK
$\mathrm{F}=$ feldspar $+\mathrm{K}$ releasing bacteria
$\mathrm{C}=$ compost $+\mathrm{N}$ fixing bacteria
$\mathrm{RP}=$ rock phosphate $+\mathrm{P}$ dissolving bacteria 
The interaction between fertilizer treatments and cultivars had significant effect on NK content of potato plants in the two seasons. The highest values of $\mathrm{N}$ content were recorded by treatments of mineral N + PK in form rocks or minerals with Lady Balfour cultivar. Meanwhile, the lowest values were obtained by all compost treatments with Lady Rosetta cultivar. On other hand, compost with $\mathrm{N}$ fixing bacteria + rocks of $\mathrm{PK}+\mathrm{P}$ dissolving and $\mathrm{K}$ releasing bacteria treatment with Lady Balfour cultivar gave the highest value of $\mathrm{K}$ content of leaves. Whereas, the lowest values were resulted by all mineral $\mathrm{N}$ treatments with Lady Rosetta.

\section{Yield components}

The data are shown in Table 7 that there were significant differences among the various treatments for yield components of potato. In both seasons, Lady Balfour cultivar significantly gave the higher values of yield and number of potato tubers than Lady Rosetta cultivar. This result was consistent with findings of Kandi et al. (2011) and Vaezzadeh et al. (2012) they indicated that the differences in yield components of potato cultivars are mainly due to difference genotype between cultivars.

Fertilizer treatments had significant effect on yield components. However, the treatment of mineral $\mathrm{N}+$ rocks of $\mathrm{PK}+\mathrm{P}$ dissolving and $\mathrm{K}$ releasing bacteria produced the highest yield and number of tubers, with no significant differences with other treatments of mineral N. On contrarily, all treatments of compost + PK in the form of minerals or rocks reduced of yield and tuber number/plant compared to mineral NPK. The lowest reductions in tuber yield/fed. were 12.2 and $10.6 \%$ by compost + rocks of PK treatment, respectively in both seasons, with no significant differences with compost + mineral PK treatment. The highest reductions of tuber yield/fed. were 18.1 and $15.4 \%$ by compost + rock $\mathrm{P}+$ mineral $\mathrm{K}$ treatment, respectively in both seasons, with no significant differences with compost + mineral $\mathrm{P}+$ rock $\mathrm{K}$ treatment. The reduction of yield by compost treatments might be due to organic fertilizer is too slow release, which leads to the nutrients available from them are insufficient for the plant requirements and thus reduce the vegetable growth, which reflected on reducing yield of tubers. These results are supported by the work of Abou-zeid et al. (2011) and Shehata et al. (2014).

Effect of interaction between fertilizer treatments and cultivars, Lady Balfour cultivar treated by mineral nitrogen treatments with the addition of PK in the form of minerals or rocks, gave the highest values of yield and number of potato tubers. The lowest values of yield and number of potato tuber were resulted from all compost treatments with Lady Rosetta cultivar. 
Table 7. Effect of treatments on yield components of two potato cultivars during 2016 and 2017 seasons

\begin{tabular}{|c|c|c|c|c|c|c|c|c|c|c|c|c|}
\hline \multirow{4}{*}{$\begin{array}{l}\text { Fertilizer } \\
\text { treatments }\end{array}$} & \multicolumn{6}{|c|}{ First season } & \multicolumn{6}{|c|}{ Second season } \\
\hline & \multicolumn{12}{|c|}{ Cultivars } \\
\hline & \multicolumn{2}{|c|}{ Rosetta } & \multicolumn{2}{|c|}{ Balfour } & \multicolumn{2}{|c|}{ Mean } & \multicolumn{2}{|c|}{ Rosetta } & \multicolumn{2}{|c|}{ Balfour } & \multicolumn{2}{|c|}{ Mean } \\
\hline & \multicolumn{12}{|c|}{ Yield (ton/fed.) } \\
\hline MNPK & 14.015 & cde & 18.280 & a & 16.160 & A & 23.700 & c & 27.010 & $a b$ & 25.390 & A \\
\hline$M N P+F$ & 13.785 & cde & 17.870 & $a$ & 15.820 & A & 23.580 & c & 26.470 & $a b$ & 25.020 & A \\
\hline$M N K+R P$ & 13.877 & cde & 17.780 & a & 15.830 & A & 23.440 & $c d$ & 26.230 & b & 24.840 & A \\
\hline$M N+R P+F$ & 14.043 & cde & 18.750 & a & 16.380 & A & 23.770 & c & 27.810 & a & 25.750 & A \\
\hline$C+$ MPK & 12.936 & ef & 14.735 & $b c$ & 13.833 & $\mathrm{BC}$ & 21.393 & ef & 23.013 & $\mathrm{~cd}$ & 22.203 & $B C$ \\
\hline$C+M P+F$ & 12.571 & $f$ & 14.364 & $b c$ & 13.467 & C & 20.910 & $f$ & 22.410 & cde & 21.660 & C \\
\hline$C+R P+M K$ & 12.271 & $f$ & 14.198 & bcd & 13.233 & C & 20.800 & $f$ & 22.173 & def & 21.487 & C \\
\hline$C+R P+F$ & 13.190 & def & 15.192 & $\mathrm{~b}$ & 14.190 & B & 21.613 & ef & 23.773 & c & 22.693 & B \\
\hline \multirow[t]{2}{*}{ Mean } & 13.337 & B & 16.397 & $A$ & & & 22.400 & B & 24.860 & $A$ & & \\
\hline & \multicolumn{12}{|c|}{ Yield (kg/plant) } \\
\hline MNPK & 0.508 & cde & 0.662 & a & 0.585 & A & 0.850 & b & 0.973 & a & 0.912 & A \\
\hline$M N P+F$ & 0.499 & cde & 0.647 & $a$ & 0.573 & A & 0.847 & bc & 0.953 & a & 0.900 & A \\
\hline MNK + RP & 0.503 & cde & 0.644 & a & 0.574 & A & 0.847 & $b c$ & 0.947 & a & 0.897 & A \\
\hline$M N+R P+F$ & 0.508 & cde & 0.679 & $a$ & 0.594 & A & 0.853 & $b$ & 0.983 & $a$ & 0.918 & A \\
\hline$C+M P K$ & 0.469 & ef & 0.534 & $b c$ & 0.501 & $\mathrm{BC}$ & 0.767 & de & 0.830 & $b c$ & 0.798 & $B C$ \\
\hline$C+M P+F$ & 0.456 & $f$ & 0.520 & bc & 0.488 & $\mathrm{BC}$ & 0.753 & e & 0.810 & bcd & 0.782 & C \\
\hline$C+R P+M K$ & 0.446 & $f$ & 0.515 & bcd & 0.480 & C & 0.747 & e & 0.797 & cde & 0.772 & C \\
\hline$C+R P+F$ & 0.478 & def & 0.550 & b & 0.514 & B & 0.777 & de & 0.857 & b & 0.817 & B \\
\hline \multirow[t]{2}{*}{ Mean } & 0.483 & B & 0.594 & A & & & 0.805 & B & 0.894 & A & & \\
\hline & \multicolumn{12}{|c|}{ Tuber number/plant } \\
\hline MNPK & 8.140 & ef & 13.334 & a & 10.737 & $A$ & 12.287 & bcd & 19.763 & a & 16.025 & A \\
\hline$M N P+F$ & 8.910 & de & 12.355 & $a b$ & 10.633 & A & 12.003 & d & 19.907 & a & 15.955 & A \\
\hline$M N K$ + RP & 8.390 & ef & 12.368 & $a b$ & 10.379 & A & 12.237 & $\mathrm{~cd}$ & 18.333 & $a$ & 15.285 & A \\
\hline$M N+R P+F$ & 8.177 & ef & 13.587 & a & 10.882 & A & 12.237 & $\mathrm{~cd}$ & 18.813 & $a$ & 15.525 & A \\
\hline $\mathrm{C}+\mathrm{MPK}$ & 7.560 & ef & 10.232 & $\mathrm{~cd}$ & 8.896 & B & 11.050 & d & 14.333 & $b c$ & 12.692 & B \\
\hline$C+M P+F$ & 7.420 & $f$ & 10.765 & c & 9.092 & B & 11.380 & d & 14.430 & $b c$ & 12.905 & B \\
\hline$C+R P+M K$ & 7.117 & $\mathrm{f}$ & 10.292 & $c d$ & 8.704 & B & 12.477 & bcd & 14.570 & b & 13.523 & B \\
\hline$C+R P+F$ & 7.290 & $\mathrm{f}$ & 11.293 & $\mathrm{bc}$ & 9.292 & $B$ & 11.140 & d & 14.570 & $b$ & 12.855 & B \\
\hline Mean & 7.875 & B & 11.778 & A & & & 11.851 & B & 16.840 & A & & \\
\hline
\end{tabular}

Means followed in same column by similar letters are not statistically different at 0.05 level according to Tukey test.

$$
\begin{aligned}
& \text { MNPK = mineral NPK } \\
& \mathrm{C}=\text { compost }+\mathrm{N} \text { fixing bacteria }
\end{aligned}
$$$$
\mathrm{F}=\text { feldspar }+\mathrm{K} \text { releasing bacteria }
$$$$
\mathrm{RP}=\text { rock phosphate }+\mathrm{P} \text { dissolving bacteria }
$$

\section{Tubers quality}

Data in Table 8 are showed effects of different fertilizers on tubers quality for two cultivars of potato. Lady Rosetta cultivar was higher than Lady Balfour cultivar in the percent of medium tubers. On the other hand, Lady Balfour cultivar produced the highest percent of small tubers. While, no significant differences were detected between both cultivars in percentage of large sizes and specific gravity of tubers. These results were noticed in the two seasons. The differences between cultivars in some properties of quality might be related to genetic factors as mentioned 
Vaezzadeh et al. (2012) and Zelelew et al. (2016). Effect of different fertilizers on tubers quality of potato, there were no significant differences among all treatments in percent of different potato sizes and specific gravity of tubers. Thus, the effect of interaction between fertilizer treatments and cultivars was not significant.

\section{Table 8. Effect of treatments on the size and specific gravity of potato tubers during 2016 and 2017 seasons}

\begin{tabular}{|c|c|c|c|c|c|c|}
\hline \multirow{4}{*}{$\begin{array}{l}\text { Fertilizer } \\
\text { treatments }\end{array}$} & \multicolumn{3}{|c|}{ First season } & \multicolumn{3}{|c|}{ Second season } \\
\hline & \multicolumn{6}{|c|}{ Cultivars } \\
\hline & Rosetta & Balfour & Mean & Rosetta & Balfour & Mean \\
\hline & \multicolumn{6}{|c|}{$\%$ Large tubers } \\
\hline MNPK & $0.00 \mathrm{a}$ & $0.00 a$ & $0.00 \mathrm{~A}$ & $2.81 \mathrm{a}$ & $1.92 \mathrm{a}$ & $2.36 \mathrm{~A}$ \\
\hline$M N P+F$ & $0.00 a$ & $0.00 \mathrm{a}$ & $0.00 \mathrm{~A}$ & $2.79 a$ & $2.10 \mathrm{a}$ & $2.45 \mathrm{~A}$ \\
\hline$M N K+R P$ & $0.00 a$ & $0.00 \mathrm{a}$ & $0.00 \mathrm{~A}$ & $2.64 a$ & $2.60 \mathrm{a}$ & $2.62 \mathrm{~A}$ \\
\hline$M N+R P+F$ & $0.00 \mathrm{a}$ & $0.00 \mathrm{a}$ & $0.00 \mathrm{~A}$ & $2.26 \mathrm{a}$ & $2.53 \mathrm{a}$ & $2.40 \mathrm{~A}$ \\
\hline$C+\mathrm{MPK}$ & $0.00 \mathrm{a}$ & $0.00 \mathrm{a}$ & $0.00 \mathrm{~A}$ & $2.59 a$ & $3.66 \mathrm{a}$ & $3.13 \mathrm{~A}$ \\
\hline$C+M P+F$ & $0.00 \mathrm{a}$ & $0.00 \mathrm{a}$ & $0.00 \mathrm{~A}$ & $2.52 \mathrm{a}$ & $3.61 \mathrm{a}$ & $3.07 \mathrm{~A}$ \\
\hline$C+R P+M K$ & $0.00 \mathrm{a}$ & $0.00 \mathrm{a}$ & $0.00 \mathrm{~A}$ & $2.00 \mathrm{a}$ & $2.97 \mathrm{a}$ & $2.48 \mathrm{~A}$ \\
\hline$C+R P+F$ & $0.00 \mathrm{a}$ & $0.00 \mathrm{a}$ & $0.00 \mathrm{~A}$ & $2.94 \mathrm{a}$ & $3.54 \mathrm{a}$ & $3.24 \mathrm{~A}$ \\
\hline \multirow[t]{2}{*}{ Mean } & $0.00 \mathrm{~A}$ & $0.00 \mathrm{~A}$ & & $2.19 \mathrm{~A}$ & $2.99 \mathrm{~A}$ & \\
\hline & \multicolumn{6}{|c|}{$\%$ Medium tubers } \\
\hline MNPK & $74.05 a$ & $58.89 b$ & $66.47 \mathrm{~A}$ & $71.31 \mathrm{a}$ & $62.40 \mathrm{~b}$ & $66.86 \mathrm{~A}$ \\
\hline$M N P+F$ & $72.61 \mathrm{a}$ & $58.36 b$ & $65.49 \mathrm{~A}$ & $72.62 a$ & $61.69 b$ & $67.16 \mathrm{~A}$ \\
\hline $\mathrm{MNK}+\mathrm{RP}$ & $73.75 \mathrm{a}$ & $61.31 b$ & $67.53 \mathrm{~A}$ & $71.83 a$ & $62.51 \mathrm{~b}$ & $67.17 \mathrm{~A}$ \\
\hline$M N+R P+F$ & $76.28 a$ & $59.14 b$ & $67.71 \mathrm{~A}$ & $71.79 a$ & $62.73 b$ & $67.26 \mathrm{~A}$ \\
\hline$C+\mathrm{MPK}$ & $70.06 a$ & $57.38 b$ & $63.72 \mathrm{~A}$ & $70.05 a$ & $63.17 b$ & $66.61 \mathrm{~A}$ \\
\hline$C+M P+F$ & $71.37 a$ & $57.75 b$ & $64.56 \mathrm{~A}$ & $70.08 \mathrm{a}$ & $62.16 b$ & $66.12 \mathrm{~A}$ \\
\hline$C+R P+M K$ & $70.11 \mathrm{a}$ & $61.29 b$ & $65.70 \mathrm{~A}$ & $71.62 a$ & $62.61 \mathrm{~b}$ & $67.12 \mathrm{~A}$ \\
\hline$C+R P+F$ & $70.53 a$ & $60.74 b$ & $65.64 \mathrm{~A}$ & $71.42 \mathrm{a}$ & $62.66 \mathrm{~b}$ & $67.04 \mathrm{~A}$ \\
\hline \multirow[t]{2}{*}{ Mean } & $72.35 \mathrm{~A}$ & $59.36 \mathrm{~B}$ & & $71.34 \mathrm{~A}$ & $62.49 \mathrm{~B}$ & \\
\hline & \multicolumn{6}{|c|}{$\%$ Small tubers } \\
\hline MNPK & $25.95 b$ & $41.11 \mathrm{a}$ & $33.53 \mathrm{~A}$ & $25.88 \mathrm{c}$ & $35.68 \mathrm{a}$ & $30.78 \mathrm{~A}$ \\
\hline$M N P+F$ & $27.39 b$ & $41.64 a$ & $34.51 \mathrm{~A}$ & $24.59 c$ & $36.21 \mathrm{a}$ & $30.40 \mathrm{~A}$ \\
\hline$M N K+R P$ & $26.25 b$ & $38.69 a$ & $32.47 \mathrm{~A}$ & $25.53 c$ & $34.89 a$ & $30.21 \mathrm{~A}$ \\
\hline$M N+R P+F$ & $23.72 b$ & $40.86 a$ & $32.29 \mathrm{~A}$ & $25.96 \mathrm{c}$ & $34.74 a$ & $30.35 \mathrm{~A}$ \\
\hline$C+\mathrm{MPK}$ & $29.94 b$ & $42.62 a$ & $36.28 \mathrm{~A}$ & $27.35 b c$ & $33.17 \mathrm{ab}$ & $30.26 \mathrm{~A}$ \\
\hline$C+M P+F$ & $28.63 b$ & $42.25 a$ & $35.44 \mathrm{~A}$ & $27.40 \mathrm{bc}$ & $34.22 \mathrm{a}$ & $30.81 \mathrm{~A}$ \\
\hline$C+R P+M K$ & $29.89 b$ & $38.71 \mathrm{a}$ & $34.30 \mathrm{~A}$ & $26.38 \mathrm{c}$ & $34.42 \mathrm{a}$ & $30.40 \mathrm{~A}$ \\
\hline$C+R P+F$ & $29.47 b$ & $39.26 a$ & $34.36 \mathrm{~A}$ & $26.30 \mathrm{c}$ & $33.80 \mathrm{ab}$ & $30.05 \mathrm{~A}$ \\
\hline \multirow[t]{2}{*}{ Mean } & $27.65 \mathrm{~B}$ & $40.64 \mathrm{~A}$ & & $26.17 \mathrm{~B}$ & $34.64 \mathrm{~A}$ & \\
\hline & \multicolumn{6}{|c|}{ Specific gravity of tuber $\left(\mathrm{g} / \mathrm{cm}^{2}\right)$} \\
\hline MNPK & $1.06 \mathrm{a}$ & $1.09 \mathrm{a}$ & $1.08 \mathrm{~A}$ & $1.07 a$ & $1.06 \mathrm{a}$ & $1.07 \mathrm{~A}$ \\
\hline$M N P+F$ & $1.09 a$ & $1.09 \mathrm{a}$ & $1.09 \mathrm{~A}$ & $1.04 \mathrm{a}$ & $1.09 \mathrm{a}$ & $1.07 \mathrm{~A}$ \\
\hline $\mathrm{MNK}+\mathrm{RP}$ & $1.06 \mathrm{a}$ & $1.06 \mathrm{a}$ & $1.06 \mathrm{~A}$ & $1.07 \mathrm{a}$ & $1.07 \mathrm{a}$ & $1.07 \mathrm{~A}$ \\
\hline$M N+R P+F$ & $1.11 \mathrm{a}$ & $1.04 \mathrm{a}$ & $1.07 \mathrm{~A}$ & $1.06 \mathrm{a}$ & $1.06 \mathrm{a}$ & $1.06 \mathrm{~A}$ \\
\hline$C+M P K$ & $1.07 \mathrm{a}$ & $1.10 \mathrm{a}$ & $1.08 \mathrm{~A}$ & $1.08 \mathrm{a}$ & $1.06 \mathrm{a}$ & $1.07 \mathrm{~A}$ \\
\hline$C+M P+F$ & $1.06 \mathrm{a}$ & $1.05 \mathrm{a}$ & $1.06 \mathrm{~A}$ & $1.06 \mathrm{a}$ & $1.04 \mathrm{a}$ & $1.05 \mathrm{~A}$ \\
\hline$C+R P+M K$ & $1.07 \mathrm{a}$ & $1.07 \mathrm{a}$ & $1.07 \mathrm{~A}$ & $1.10 \mathrm{a}$ & $1.05 \mathrm{a}$ & $1.08 \mathrm{~A}$ \\
\hline$C+R P+F$ & $1.07 \mathrm{a}$ & $1.07 \mathrm{a}$ & $1.07 \mathrm{~A}$ & $1.10 \mathrm{a}$ & $1.07 \mathrm{a}$ & $1.09 \mathrm{~A}$ \\
\hline Mean & $1.07 \mathrm{~A}$ & $1.07 \mathrm{~A}$ & & $1.07 \mathrm{~A}$ & $1.06 \mathrm{~A}$ & \\
\hline \multicolumn{7}{|c|}{$\begin{array}{l}\text { Means followed in same column by similar letters are not statistically different at } 0.05 \text { level according to Tuk } \\
\text { test. }\end{array}$} \\
\hline $\begin{array}{l}\text { test. } \\
\text { MNPK = mine } \\
\mathrm{C}=\text { compost }\end{array}$ & xing bact & & $\begin{array}{l}F=\text { fel } \\
R P=\end{array}$ & hosphat & $\begin{array}{l}\text { bacteria } \\
\text { dissolving }\end{array}$ & \\
\hline
\end{tabular}




\section{Tuber compositions}

Data pertaining to the effect of the replacement of mineral fertilizers by natural ones on potato tuber compositions are tabulated in Table 9 and 10. The tubers of Lady Rosetta cultivar were higher in contents of TSS, dry mater, starch and carbohydrate than Lady Balfour tubers. While, Lady Balfour tubers were higher in nitrate content. Superiority of Lady Rosetta cultivar in these traits may be due to genotype for cultivar. These results are supported by Yaghbani and Mohammadzadeh (2005), they reported that there was significant difference in dry matter starch and carbohydrate contents between different cultivars of potato. Since starch forms 60$80 \%$ of dry matter, this makes a positive correlation between starch content and dry matter of potatoes. In addition, starch is the predominant component of carbohydrates in potatoes so potato cultivars that contain high dry matter, also contain high starch and carbohydrates as reported by Kandi et al. (2011).

Table 9. Effect of treatments on TSS and dry matter of potato tubers during 2016 and 2017 seasons

\begin{tabular}{|c|c|c|c|c|c|c|}
\hline \multirow{4}{*}{$\begin{array}{l}\text { Fertilizer } \\
\text { treatments }\end{array}$} & \multicolumn{3}{|c|}{ First season } & \multicolumn{3}{|c|}{ Second season } \\
\hline & \multicolumn{6}{|c|}{ Cultivars } \\
\hline & Rosetta & Balfour & Mean & Rosetta & Balfour & Mean \\
\hline & \multicolumn{6}{|c|}{ TSS in tuber $(\%)$} \\
\hline MNPK & $7.22 \mathrm{a}$ & $4.31 \mathrm{~b}$ & $5.77 \mathrm{~A}$ & $6.10 \mathrm{a}$ & $4.57 b$ & $5.33 \mathrm{~A}$ \\
\hline $\mathrm{MNP}+\mathrm{F}$ & $7.20 \mathrm{a}$ & $4.38 \mathrm{~b}$ & $5.79 \mathrm{~A}$ & $6.13 \mathrm{a}$ & $4.27 b$ & $5.20 \mathrm{~A}$ \\
\hline MNK + RP & $7.45 a$ & $4.51 \mathrm{~b}$ & $5.98 \mathrm{~A}$ & $6.63 a$ & $4.23 b$ & $5.43 \mathrm{~A}$ \\
\hline$M N+R P+F$ & $7.10 \mathrm{a}$ & $4.18 b$ & $5.64 \mathrm{~A}$ & $6.13 \mathrm{a}$ & $4.27 b$ & $5.20 \mathrm{~A}$ \\
\hline$C+M P K$ & $7.47 a$ & $4.65 b$ & $6.06 \mathrm{~A}$ & $5.93 \mathrm{a}$ & $4.20 \mathrm{~b}$ & $5.07 \mathrm{~A}$ \\
\hline$C+M P+F$ & $7.24 a$ & $4.41 \mathrm{~b}$ & $5.83 \mathrm{~A}$ & $6.40 \mathrm{a}$ & $4.10 \mathrm{~b}$ & $5.25 \mathrm{~A}$ \\
\hline$C+R P+M K$ & $7.50 \mathrm{a}$ & $4.44 b$ & $5.97 \mathrm{~A}$ & $6.27 \mathrm{a}$ & $3.97 b$ & $5.12 \mathrm{~A}$ \\
\hline$C+R P+F$ & $7.50 \mathrm{a}$ & $4.14 \mathrm{~b}$ & $5.82 \mathrm{~A}$ & $6.13 \mathrm{a}$ & $4.07 \mathrm{~b}$ & $5.10 \mathrm{~A}$ \\
\hline \multirow[t]{2}{*}{ Mean } & $7.33 \mathrm{~A}$ & $4.38 \mathrm{~B}$ & & $6.22 \mathrm{~A}$ & $4.21 \mathrm{~B}$ & \\
\hline & \multicolumn{6}{|c|}{ Dry matter in tuber (\%) } \\
\hline MNPK & $22.48 \mathrm{C}$ & $17.90 \mathrm{f}$ & $20.19 \mathrm{C}$ & $21.67 \mathrm{~cd}$ & $16.43 \mathrm{e}$ & $19.05 \mathrm{CD}$ \\
\hline MNP + F & $22.60 \mathrm{bc}$ & $18.16 \mathrm{ef}$ & $20.38 \mathrm{C}$ & $21.87 \mathrm{bcd}$ & $16.70 \mathrm{e}$ & $19.28 \mathrm{BCD}$ \\
\hline$M N K+R P$ & $22.62 b c$ & 18.53 def & $20.58 \mathrm{C}$ & $20.77 \mathrm{~d}$ & $16.40 \mathrm{e}$ & $18.58 \mathrm{D}$ \\
\hline$M N+R P+F$ & $22.91 \mathrm{bc}$ & 18.81 def & $20.86 \mathrm{BC}$ & $22.37 \mathrm{bc}$ & $16.83 \mathrm{e}$ & $19.60 \mathrm{BC}$ \\
\hline$C+M P K$ & $22.99 \mathrm{bc}$ & 19.00 def & $20.99 B C$ & $22.97 a b$ & $16.90 \mathrm{e}$ & $19.93 \mathrm{AB}$ \\
\hline$C+M P+F$ & $23.74 a b$ & $19.16 \mathrm{de}$ & $21.45 \mathrm{AB}$ & $23.07 a b$ & $16.90 \mathrm{e}$ & $19.98 \mathrm{AB}$ \\
\hline$C+R P+M K$ & $23.63 a b c$ & 19.23 de & $21.43 \mathrm{AB}$ & $23.73 a$ & $17.07 \mathrm{e}$ & $20.40 \mathrm{~A}$ \\
\hline$C+R P+F$ & $24.44 a$ & $19.63 \mathrm{~d}$ & $22.03 \mathrm{~A}$ & $23.83 \mathrm{a}$ & $17.07 \mathrm{e}$ & $20.45 \mathrm{~A}$ \\
\hline Mean & $23.18 \mathrm{~A}$ & $18.80 \mathrm{~B}$ & & $22.53 \mathrm{~A}$ & $16.79 \mathrm{~B}$ & \\
\hline \multicolumn{7}{|c|}{$\begin{array}{l}\text { Means followed in same column by similar letters are not statistically different at } 0.05 \text { level according to } \\
\text { Tukey test. }\end{array}$} \\
\hline $\begin{array}{l}\text { MNPK = miner } \\
\mathrm{C}=\text { compost }\end{array}$ & bacteria & & $\begin{array}{l}F=\text { feldsp } \\
R P=\text { roc }\end{array}$ & $\begin{array}{l}\text { K releasing } \\
\text { sphate }+\mathrm{P}\end{array}$ & $\begin{array}{l}\text { eria } \\
\text { Iving bac }\end{array}$ & \\
\hline
\end{tabular}

Effect of different fertilizers on potato tuber contents, there were no significant differences among all treatments in tuber content of TSS. All treatments of compost + rocks of $P$ and $K$ individual or in combinations increased tuber contents of dry matter, starch and carbohydrate compared to mineral NPK. In contrast, these treatments decreased nitrate content in tuber. The superiority of compost treatments over mineral fertilizer in dry matter, starch and carbohydrate in tubers may be due to the degradation of organic 
matter and the releasing $\mathrm{CO}_{2}$ in soil (Jarvan and Edesi, 2009). It may also be due to available nitrogen in organic fertilizer is low, which leads to metabolism more toward to formation of carbon compounds, such as starch and cellulose (Rembialkowska, 2007). These results were in accordance with those obtained by Abdel-Salam \& Shams (2012) and Shehata et al. (2014). Using compost + rocks of PK treatment led to reduction $45 \%$ of nitrate content in tuber compared to mineral NPK treatment as average in both seasons. This is very important, because nitrate can easily converted into nitrite, which causes methaemoglobinaemia illness especially in children. Moreover, nitrite can react with amines to create nitrosamines, which are carcinogenic (Mahmoud et al., 2009). This was attributed to the supply of readily available nitrate from mineral $\mathrm{N}$ fertilizers to the plants while, in the organic $\mathrm{N}$ treatments, nitrate release was comparatively slow. The nitrate concentrations of tubers increased with mineral $\mathrm{N}$ fertilizers. Similar finding was obtained by Mohammadi et al. (2013) and El-Sayed et al. (2015)

Table 10. Effect of treatments on starch, carbohydrate and nitrate in dry matter of potato tubers during 2016 and 2017 seasons

\begin{tabular}{|c|c|c|c|c|c|c|}
\hline \multirow{4}{*}{$\begin{array}{l}\text { Fertilizer } \\
\text { treatments }\end{array}$} & \multicolumn{3}{|c|}{ First season } & \multicolumn{3}{|c|}{ Second season } \\
\hline & \multicolumn{6}{|c|}{ Cultivars } \\
\hline & Rosetta & Balfour & Mean & Rosetta & Balfour & Mean \\
\hline & \multicolumn{6}{|c|}{ \% Starch } \\
\hline MNPK & $75.52 b c$ & $71.23 \mathrm{ef}$ & $73.37 \mathrm{~B}$ & $74.90 \mathrm{~b}$ & $70.50 \mathrm{f}$ & $72.70 \mathrm{~B}$ \\
\hline$M N P+F$ & 75.58 bc & $71.21 \mathrm{ef}$ & $73.39 \mathrm{~B}$ & $74.83 b c$ & $70.53 \mathrm{f}$ & $72.68 \mathrm{~B}$ \\
\hline$M N K+R P$ & $75.66 b$ & $71.15 f$ & $73.41 \mathrm{~B}$ & $74.90 \mathrm{~b}$ & $70.47 \mathrm{f}$ & $72.68 \mathrm{~B}$ \\
\hline$M N+R P+F$ & $75.60 \mathrm{bc}$ & 71.29 ef & $73.45 \mathrm{~B}$ & $74.87 b c$ & $70.63 \mathrm{ef}$ & $72.75 \mathrm{~B}$ \\
\hline$C+$ MPK & $77.20 \mathrm{ab}$ & 72.86 de & $75.03 \mathrm{~A}$ & $76.43 a b$ & $72.20 \mathrm{de}$ & $74.32 \mathrm{~A}$ \\
\hline$C+M P+F$ & $77.66 a$ & $73.81 \mathrm{~d}$ & $75.74 \mathrm{~A}$ & $76.87 a$ & $73.13 d$ & $75.00 \mathrm{~A}$ \\
\hline$C+R P+M K$ & $77.66 a$ & $73.75 d$ & $75.71 \mathrm{~A}$ & $76.85 a$ & $73.03 d$ & $74.94 \mathrm{~A}$ \\
\hline$C+R P+F$ & $77.65 a$ & $73.91 \mathrm{~cd}$ & $75.78 \mathrm{~A}$ & $76.87 \mathrm{a}$ & $73.23 \mathrm{~cd}$ & $75.05 \mathrm{~A}$ \\
\hline \multirow[t]{2}{*}{ Mean } & $76.57 \mathrm{~A}$ & $72.40 \mathrm{~B}$ & & $75.81 \mathrm{~A}$ & $71.72 \mathrm{~B}$ & \\
\hline & \multicolumn{6}{|c|}{$\%$ Carbohydrate } \\
\hline MNPK & 84.60 bc & 79.79 ef & $82.20 \mathrm{~B}$ & $83.82 b$ & $78.89 \mathrm{f}$ & $81.36 \mathrm{~B}$ \\
\hline$M N P+F$ & $84.67 b c$ & 79.77 ef & $82.22 \mathrm{~B}$ & $83.74 b c$ & $78.94 \mathrm{f}$ & 81.34 B \\
\hline $\mathrm{MNK}+\mathrm{RP}$ & $84.76 b$ & $79.71 \mathrm{f}$ & $82.24 B$ & $83.84 b$ & $78.88 \mathrm{f}$ & $81.36 \mathrm{~B}$ \\
\hline$M N+R P+F$ & 84.70 bc & 79.87 ef & $82.28 \mathrm{~B}$ & $83.77 b c$ & 79.03 ef & $81.40 \mathrm{~B}$ \\
\hline$C+M P K$ & $86.49 a b$ & 81.62 de & $84.06 \mathrm{~A}$ & $85.52 a b$ & $80.77 \mathrm{de}$ & $83.15 \mathrm{~A}$ \\
\hline$C+M P+F$ & $87.00 \mathrm{a}$ & $82.69 \mathrm{~d}$ & $84.85 \mathrm{~A}$ & $86.02 \mathrm{a}$ & $81.83 \mathrm{~d}$ & $83.93 \mathrm{~A}$ \\
\hline$C+R P+M K$ & $86.99 a$ & $82.62 d$ & $84.81 \mathrm{~A}$ & $86.02 a$ & $81.76 \mathrm{~d}$ & $83.89 \mathrm{~A}$ \\
\hline$C+R P+F$ & $86.99 a$ & $82.80 \mathrm{~cd}$ & $84.90 \mathrm{~A}$ & $86.02 a$ & $81.94 \mathrm{~cd}$ & $83.98 \mathrm{~A}$ \\
\hline \multirow[t]{2}{*}{ Mean } & $85.78 \mathrm{~A}$ & $81.11 \mathrm{~B}$ & & $75.81 \mathrm{~A}$ & $80.26 \mathrm{~B}$ & \\
\hline & \multicolumn{6}{|c|}{$\%$ Nitrate } \\
\hline MNPK & $0.320 \mathrm{~b}$ & $0.398 a$ & $0.359 \mathrm{~A}$ & $0.284 b$ & $0.361 \mathrm{a}$ & $0.322 \mathrm{~A}$ \\
\hline$M N P+F$ & $0.314 b$ & $0.392 a$ & $0.353 \mathrm{~A}$ & $0.278 b$ & $0.359 a$ & $0.319 \mathrm{~A}$ \\
\hline$M N K+R P$ & $0.316 b$ & $0.402 a$ & $0.359 \mathrm{~A}$ & $0.280 \mathrm{~b}$ & $0.365 a$ & $0.323 \mathrm{~A}$ \\
\hline$M N+R P+F$ & $0.323 b$ & $0.398 \mathrm{a}$ & $0.361 \mathrm{~A}$ & $0.282 b$ & $0.361 \mathrm{a}$ & $0.322 \mathrm{~A}$ \\
\hline $\mathrm{C}+\mathrm{MPK}$ & $0.179 d$ & $0.221 \mathrm{c}$ & $0.200 \mathrm{~B}$ & $0.154 c$ & $0.194 \mathrm{c}$ & $0.174 \mathrm{~B}$ \\
\hline$C+M P+F$ & $0.179 d$ & $0.216 \mathrm{c}$ & $0.197 \mathrm{~B}$ & $0.154 \mathrm{c}$ & $0.189 \mathrm{c}$ & $0.171 \mathrm{~B}$ \\
\hline$C+R P+M K$ & $0.188 d$ & $0.215 c$ & $0.201 B$ & $0.163 c$ & $0.188 \mathrm{c}$ & $0.175 \mathrm{~B}$ \\
\hline$C+R P+F$ & $0.187 \mathrm{~d}$ & $0.213 c$ & $0.200 \mathrm{~B}$ & $0.162 c$ & $0.188 \mathrm{C}$ & $0.175 \mathrm{~B}$ \\
\hline Mean & $0.251 \mathrm{~B}$ & $0.307 \mathrm{~A}$ & & $0.220 \mathrm{~B}$ & $0.276 \mathrm{~A}$ & \\
\hline
\end{tabular}

Means followed in same column by similar letters are not statistically different at 0.05 level according to Tukey test. MNPK = mineral NPK $\mathrm{F}=$ feldspar $+\mathrm{K}$ releasing bacteria $\mathrm{C}=$ compost $+\mathrm{N}$ fixing bacteria $\mathrm{RP}=$ rock phosphate $+\mathrm{P}$ dissolving bacteria 
The interaction between fertilizer treatments and cultivars had a significant effect on potato tuber contents in both seasons except TSS, which showed no significant differences among all treatments. The highest contents of dry matter, starch and carbohydrate in tubers were recorded by all compost treatments with Lady Rosetta cultivar. On other hand, all mineral $\mathrm{N}$ treatments with Lady Balfour gave the highest nitrate content in tuber.

\section{Economic evaluate}

The economic evaluation in Table 11 clearly indicated that although Lady Balfour cultivar was superior to Lady Rosetta cultivar in the yield quantity, Lady Rosetta was higher than Lady Balfour in the gross and net return. This is attributed that the sale price of Lady Rosetta tubers was higher than the Lady Balfour tubers. Concerning the treatments, using mineral $\mathrm{N}+$ rocks of $\mathrm{PK}+\mathrm{P}$ dissolving and $\mathrm{K}$ releasing bacteria recorded the highest gross and net return. This may be due to the superiority of this treatment to other treatments in the yield quantity. In regard the interaction between cultivars and fertilizer treatments, Lady Balfour cultivar with application of mineral $\mathrm{N}+$ rocks of $\mathrm{PK}+\mathrm{P}$ dissolving and $\mathrm{K}$ releasing bacteria gave the highest gross and net return. While, Lady Balfour cultivar with using $C+R P+M K$ gave the lowest gross and net return. It can be noted that Lady Balfour cultivar with all the treatments of nitrogen mineral fertilizer recorded higher net returns than Lady Rosetta for the same treatments. Conversely, Lady Rosetta was superior to Lady Balfour with same compost treatments.

Table 11. Economic evaluate of different treatments as gross return and net return of two potato cultivars as mean for both seasons (2016 and 2017)

\begin{tabular}{|c|c|c|c|c|c|c|c|c|c|}
\hline \multirow{2}{*}{ Treatments } & \multirow{2}{*}{$\begin{array}{c}\text { Total cost } \\
(\text { L.E./fed.) }\end{array}$} & \multicolumn{2}{|c|}{ Yield (ton/fed.) } & \multicolumn{3}{|c|}{ Gross return (L.E./fed.) } & \multicolumn{3}{|c|}{ Net return (L.E./fed.) } \\
\hline & & Rosseta & Balfour & Rosseta & Balfour & Mean & Rosseta & Balfour & Mean \\
\hline MNPK & 18547 & 18.857 & 22.645 & 37715 & 38497 & 38106 & 19168 & 19949 & 19559 \\
\hline$M N P+F$ & 17815 & 18.682 & 22.170 & 37365 & 37689 & 37527 & 19550 & 19874 & 19712 \\
\hline$M N K+R P$ & 18384 & 18.658 & 22.005 & 37317 & 37409 & 37363 & 18933 & 19025 & 18979 \\
\hline $\begin{array}{l}\mathrm{MN}+\mathrm{RP}+ \\
\mathrm{F}\end{array}$ & 17652 & 18.906 & 23.280 & 37813 & 39576 & 38694 & 20161 & 21924 & 21042 \\
\hline$C+M P K$ & 20603 & 17.165 & 18.874 & 34330 & 32086 & 33208 & 13727 & 11483 & 12605 \\
\hline$C+M P+F$ & 19871 & 16.741 & 18.387 & 33481 & 31258 & 32370 & 13610 & 11387 & 12499 \\
\hline $\begin{array}{l}\mathrm{C}+\mathrm{RP}+ \\
\mathrm{MK}\end{array}$ & 20440 & 16.535 & 18.186 & 33071 & 30916 & 31993 & 12631 & 10476 & 11553 \\
\hline$C+R P+F$ & 19708 & 17.402 & 19.483 & 34803 & 33121 & 33962 & 15095 & 13413 & 14254 \\
\hline Mean & & 17.868 & 20.629 & 35737 & 35069 & & 16609 & 15941 & \\
\hline
\end{tabular}

Sale price of Lady Rosseta tubers $=2000$ L.E./ton

Sale price of Lady Balfour tubers $=1700$ L.E. $/$ ton

\section{CONCLUTION}

It could be concluded that rock phosphate and feldspar with phosphate dissolving bacteria and $\mathrm{K}$ releasing bacteria could be an alternatives to mineral PK for similar yield and 
quality of potatoes, as well as obtain the highest net return. While, compost with $\mathrm{N}$ fixing bacteria could be an alternative to mineral $\mathrm{N}$ obtain slightly low yield of potatoes but it's distinguished by high quality and healthy.

\section{ACKNOWLEDGEMENT}

This work has been supported by Central Lab of Organic Agriculture, Agriculture Research Center - Horticulture Departments, Faculty of Agriculture, Minia University.

\section{REFERENCES}

1. Abdel-Mouty, M. M. and N. H. M. El-Greadly, 2008. The productivity of two okra cultivars as affected by gibberellic acid, organic $\mathrm{N}$, rock phosphate and feldspar application. Journal of Applied Sciences Research, 4(6): 627-636.

2. Abd-El-Salam, M. A. and A.S. Shams, 2012. Feldspar-K fertilization of potato (Solanum tuberosum L.) augmented by biofertilizer. American-Eurasian J. Agric. \& Environ. Sci., 12(6): 694-699.

3. Abou-Hussein, S. D., 2005. Yield and quality of potato crop as affected by the application rate of potassium and compost in sandy soil. Ann. Agric. Sci., Ain Shams Univ., Cairo, 50(2): 573-586.

4. Abou-zeid, Y. M and M. A. Bakry, 2011. Integrated effect of bio-organic manures and mineral fertilizers on potato productivity and the fertility status of a calcareous soil. Aust. J. Basic App. Sci., 5(8): 1385-1399.

5. Adams, M. J. and G. A. Hide, 1981. Effects of common scab (Streptomyces scabies) on potatoes. Ann. App. Biol., 98(2): 211-216.

6. Al-Moshileh, A. M., K. N. Al-Redhaiman, and M. Z. El-Shinawy, 2004. The effect of nitrogen sources on yield and nitrate accumulation in lettuce and cabbage plants. Egypt. J. Appl. Sci., 19: 646-654.

7. AOAC, 2005. Official methods of analysis. 15th ed. Association of Official Agricultural Chemists. 12th Ed., Washengton, D.C., USA.

8. Cottenie, A., M. Verloo, L. Kiekers, G. Velghe and R. Camrbynek 1982. Chemical Analysis of Plants and Soils. Hand Book, 1-63, Ghent, Belgium.

9. El-Sayed, S. F., H. A. Hassan, and M. M. El-Mogy, 2015. Impact of bio- and organic fertilizers on potato yield, quality and tuber weight loss after harvest. Potato Research, 58 : 67-81.

10. El-Sayed, S. F., H. A. Hassan, M. M. El-Mogy and A. Abdel-Wahab, 2014. Growth, yield and nutrient concentration of potato plants grown under organic and conventional fertilizer systems. American-Eurasian J. Agric. \& Environ. Sci., 14 (7): 636-643.

11. FAO 1980. Soil and Plant Analysis. Soils Bulletin 38/2,250P.

12. FAO, 2014. FAOSTAT/Statistical Database.

13. Fiorentino, N. and M. Fagnano 2011. Soil fertilization with composted solid waste: short term effects on lettuce production and mineral $\mathrm{N}$ availability. Geophysical Research Abstracts, Vol. 13, p 10520.

14. General Authority for the Control of Exports and Imports, 2015. Monthly e-newsletter of the Council, Published by Agricultural Export Council, issue 22.

15. Jarvan, M. and L. Edesi, 2009. The effect of cultivation methods on the yield and biological quality of potato. Agronomy Research 7(Special issue I): 289-299. 
16. Kandi, M. A. S., A. Tobeh, A. Gholipoor, S. Jahanbakhsh, D. Hassanpanah and O. Sofalian, 2011. Effects of different $\mathrm{N}$ fertilizer rate on starch percentage, soluble sugar, dry matter, yield and yield components of potato cultivars. Australian Journal of Basic and Applied Sciences, 5(9): 1846-1851.

17. Labib, B. F., T. K. Ghabour, I. S. Rahim and M. M. Wahba, 2012. Effect of potassium bearing rock on the growth and quality of potato crop (Solanum tuberosum). J. Agric. Biotech. Sustainable Dev., 4(1): 7-15.

18. Lairon, D. 2009. Nutritional quality and safety of organic food a review. Agronomy for Sustainable Development, 30(1): 33-41.

19. Lee, J. Y. and S. H. Song, 2007. Evaluation of ground water quality in coastal areas, implications for sustainable agriculture. Environ. Geol., 52(7): 1231-1242.

20. Mahmoud, E. K., N. Abd EL-Kader, P. Robin, N. Akkal-Corfini and L. Abd El-Rahman, 2009. Effects of different organic and inorganic fertilizers on Cucumber Yield and Some Soil Properties. World J. Agric.Sci., 5(4): 408-414.

21. Mashhoor, W. A, M. A. El-Borollosy, H. H. A.Abdel-Azeem, S. A. Nasr and S. M. Selim, 2002. Biofertilization of wheat plants exposed to environmental conditions. J. Agric. Sci, Ain Shams Univ., 10(2): 543-565.

22. Ministry of Agriculture and Land Reclamation (EAS), Economic Affairs Sector 2015. The Indicators Agriculture Statistics.

23. Mohammadi, G. R., A. R. Ajirloo, M. E. Ghobadi and A. Najaphy, 2013. Effects of nonchemical and chemical fertilizers on potato (Solanum tuberosum L.) yield and quality. Journal of Medicinal Plants Research, 7(1): 36-42.

24. Rembialkowska, E., 2007. Quality of plant products from organic agriculture. J Sci Food Agric., 87:2757-2762.

25. Shaaban, L. D., K. E. Khalil, and R. E. El-Tantawy, 2015. Integration effect between biofertilizers and natural fertilizers on growth, NPK uptake and yield parameters of wheat plant grown in alkaline soil. Egypt. J. Bot., 55(2): 269-279.

26. Shehata S. A., M. A. El-Helaly and M. A. El-Said, 2014. Using natural alternative fertilizers for potato production under sandy soil condtions in Egypt. J. Plant Production, Mansoura Univ., 5(10): 1745-1757.

27. Sheng X. F., L.Y. He and W. Y. Huang, 2002. The conditions of releasing potassium by a silicate dissolving bacterial strain NBT. Agr. Sci. China, 1: 662-666.

28. Snedecor, G. W. and W. G. Cochran 1980. Statistical methods. Sixth Edition, Iowa state university press, Ames., Iowa, U.S.A.

29. Vaezzadeh, M. and M. Naderidarbaghshahi, 2012. The Effect of various nitrogen fertilizer amounts on yield and nitrate accumulation in tubers of two potato cultivars in cold regions of Isfahan (Iran). International Journal of Agriculture and Crop Sciences, 4: 1688-1691.

30. Yaghbani, M. and J. Mohammadzadeh, 2005. Study of starch physic-chemical characteristics in potato dominant cultivars in Golesten region. Iranian Journal of Nutrition Sciences and Food Technology, 2(4): 71-79.

31. Zelelew, D. Z., S. Lal, T. T. Kidane and M. G. Biniam, 2016. Effect of potassium levels on growth and productivity of potato varieties. American Journal of Plant Sciences, 7: 16291638. 


\title{
إنتاج البطاطس عن طريق الاضافات الطبيعية و العضوية والحيوية
}

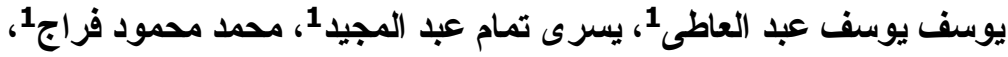 \\ سعد أبو الحسن عبد العزيز2، باسم عبد الناصر عبد المجيد سالم1 1
}

$$
\begin{aligned}
& \text { 1. قسم البساتين ـ كلية الزراعة ـ جامعة المنيا- مصر }
\end{aligned}
$$

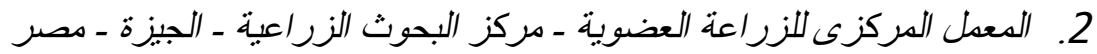

أجريت تجربة حقلية لتقييم ما إذا كان استخدام الكمبوست و الصخور الطبيعية من الفوسفات و البوتاسيوم مع الأسمدة الحيوية يمكن أن تحل محل الأسمدة المعدنية في إنتاج البطاطس. أجريت

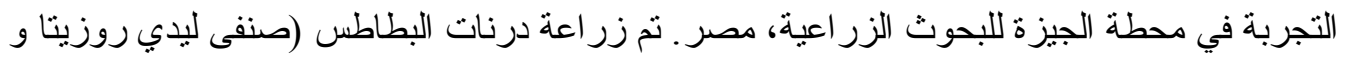

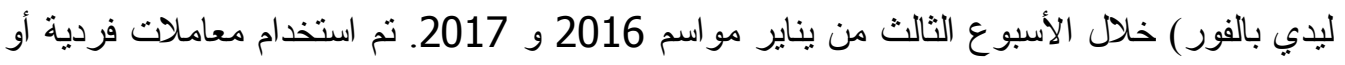

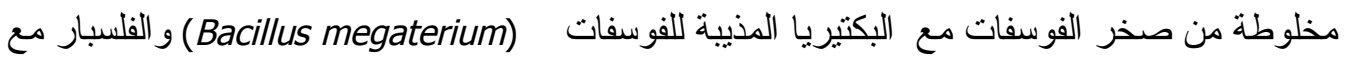

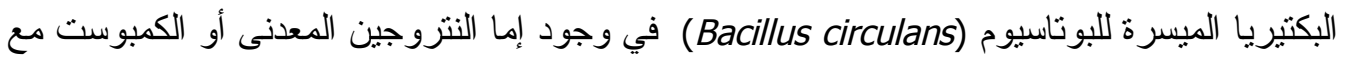

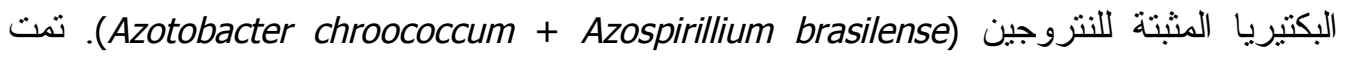
مقارنة تأثير هذه المعاملات على خصائص النمو ومحتوى النباتات من العناصر الغذائية ومكونات

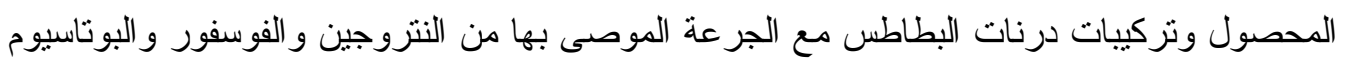
كأسمدة معدنية.

اثشارت النتائج الى ان الصنف ليدى بالفور كان أفضل معنوياً في معظم الصفات الخضرية

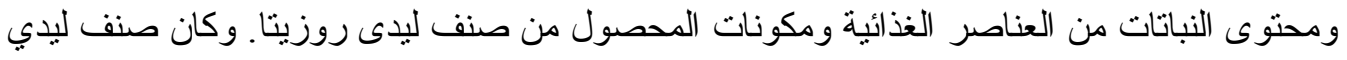

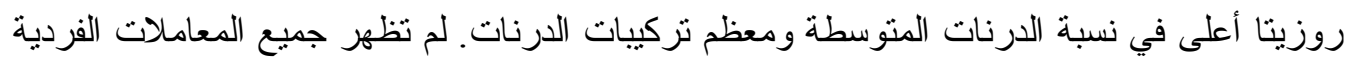

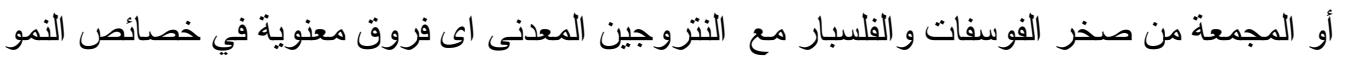

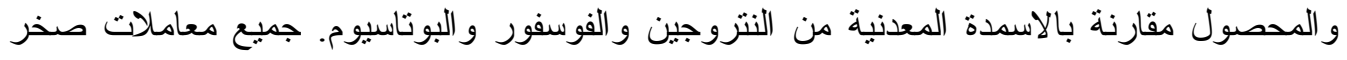

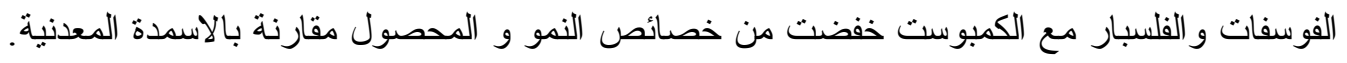

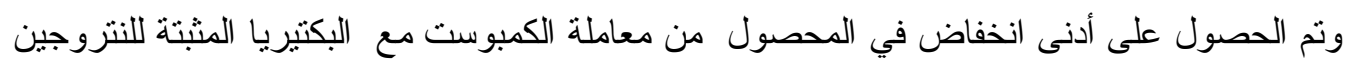

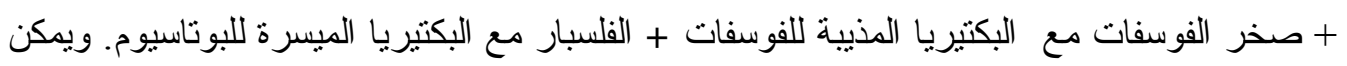

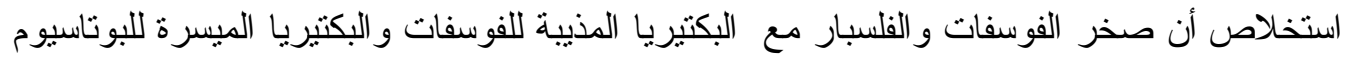

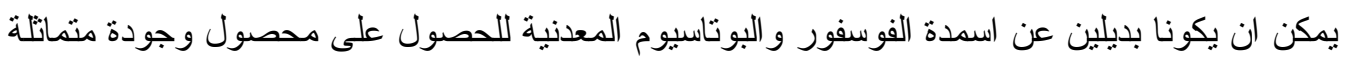
من البطاطس وكذلك الحصول على أعلى صافى عائد. يمكن أن يكون الكمبوست مع البكان البكتيريا المثبتة

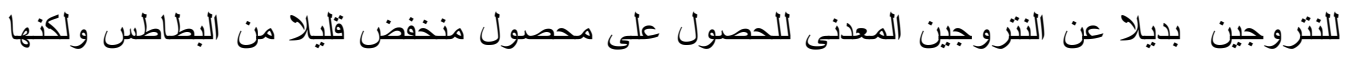
تتمييز بالجودة العالية وصحية. 
PRODUCING POTATO VIA NATURAL, ORGANIC AND BIO AMENDMENTS 